\title{
Developing Educational Smart Grid Laboratory for the UAEU
}

http://dx.doi.org/10.3991/ijoe.v10i2.3170

\author{
A. M. Gaouda, Ahmed Abd-Rabou and Abdulrahman Dahir \\ UAE University, Al Ain, UAE
}

\begin{abstract}
The goal of this paper is to present the first stage of developing an educational laboratory to teach smart grid design and implementations for both under graduate and graduate levels. Hampden 180 simulator that represents conventional generation, transmission, and distribution power system is integrated with intelligent electronic devices and tied together in an architecture that provides reliable and fault-tolerant protection, control and monitoring. The upgraded laboratory will demonstrate high level of functionality and satisfying the design and operation objectives of smart grid metering, protection, control, monitoring and communication. Part 2 of this paper will discuss and present the implementation stage.
\end{abstract}

Index Terms-smart grid, intelligent electronic devices, wireless sensors, power simulator.

\section{INTRODUCTION}

Transforming conventional power systems towards intelligent systems has led to engineering initiatives known as 'smart grid', 'intelligrid', "gridwise", "modern grid", "perfect grid", "future grid" [1]. Essential issues of Smart Grid (SG) concept are the addition of intelligent and twoway digital communication to the electric power grid to improve system efficiency, reliability and security as well as the expansion of the grid infrastructure to accommodate renewable resources, energy storage and microgrids. Furthermore, the penetration of information technology that allow full digital control and development of new applications will impact the innovation level of electricity uses for rapid outage detection, real-time pricing feedback to end-users and better routing of power and demand management. Increasing the efficiency of energy delivery, enhancing the reliability of the power system, and mitigating the adverse impact of conventional fuel plants on the environment can all be achieved through increases in the intelligence level of power systems [1-5].

Power engineering education suitable for the smart grid represents a challenging task to overcome the shortage of workforce with the integrated knowledge in different areas of smart grid and the required teaching approaches to develop the integrated skills needed by smart grid engineers $[1,4]$. Demonstrating the integration complexity at different levels is an open challenge [4]. The lack of interaction in the simulation and modeling tools that integrates power and communication systems with their related control was reported in $[6,7]$.

This leads to the objective of this paper in developing the knowledge needed to understand the concepts and overcome the challenges of teaching of the smart grid. The first stage of this paper is the upgrading stage of the existing power system simulator in the UAEU (United Arab Emirates University) power system laboratory. The second stage will present samples of implementations to overcome the challenges of teaching smart grid. The primary purpose for upgrading this lab is to satisfy the university goals of increasing the engineering hands on experience and enhancing the research capabilities of UAEU students. Moreover, data storing and communication capability of the proposed equipment will be utilized to satisfy the COE (College of Engineering) goals of redesigning our interactive courses utilizing IT (Information Technology) capability and web-based elements to cater for different learning modes of students so as to increase their comprehension of subjects covered.

The paper is organized as follows. Section II discusses program assessment at the UAEU and introduces smart grid education, main features of the proposed laboratory and the objectives of the upgrading. Bloom's Taxonomy will be discussed. The main elements of the existing Hampden 180 Power System Simulator (PSS) are presented in section III. Different generation scenarios, operation options, system configuration and possible simulated faults are illustrated. Upgrading of the power system simulator is presented in Section IV. A list of IEDs (Intelligent Electronic Devices) integrated with the simulator and their functions that support smart grid implementations are discussed. Educational outcomes are briefly introduced in Section V. A Conclusion is presented in section VI.

\section{UAEU AND SMART GRID EDUCATION}

The College of Engineering at the UAEU adopted an engineering curriculum that fosters innovation and better prepare graduates to effectively meet current and future professional needs as well as rapid technological advances encountered worldwide. The UAEU engineering curriculum focuses on a balance of knowledge and skills that prepare graduates to analyze and design engineering systems and become technical leaders in their fields, and provides students multiple opportunities to demonstrate these skills through laboratory hands-on experience, project based learning relevant to their disciplines, design challenges, and professional activities.

The Electrical Engineering undergraduate program is accredited by the Accreditation Board for Engineering and Technology (ABET). The program assessment and evaluation process is presented in Fig. 1. The assessment measures are based on students' graduation projects, course assessment, exit interview, alumni survey, employer survey and internship Industrial survey. 


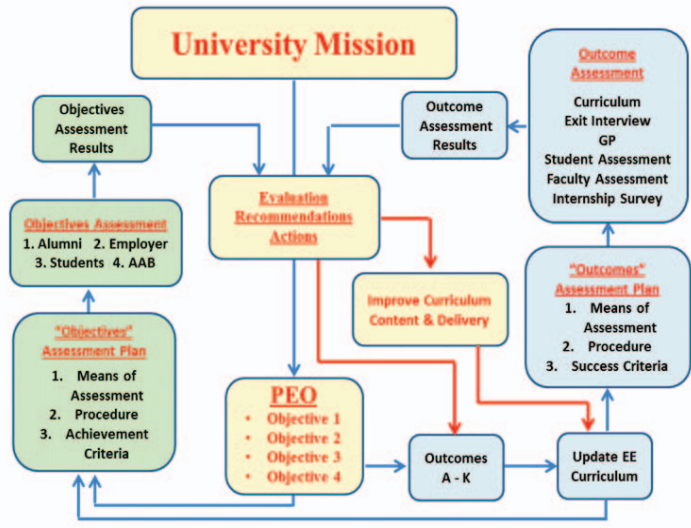

Figure 1. The program assessment and evaluation process

The integration and application of real-time monitoring, advanced sensing, communications, analytics, and control, enabling the dynamic flow of both energy and information to accommodate existing and new forms of supply, delivery, and use in a secure, reliable, and efficient electric power system, from generation source to end-user is the goal of smart grids.

This goal that approaches generation, transmission, distribution and utilization systems design and operation requires an integrative strategy. Besides the knowledge of power systems, several areas such as communications, controls, electronics, instrumentation, electromagnetics, computers and IT must be brought in the design and operation $[1,4]$. This multidisciplinary and integrative nature makes the smart grid education more challenging as compared with traditional power system education [8, 9]. Hence additional topics and tools need to be developed to facilitate the learning process and develop experiments with physical integrated solutions that allow future smart grid designer and operators to gain knowledge at different levels as mentioned in Bloom's Taxonomy $[8,9]$.

Bloom's Taxonomy defines six different levels of thinking from basic to higher levels of critical thinking skills: Remembering is retrieving, recognizing, and recalling relevant knowledge from long-term memory. Understanding is constructing meaning from oral, written, and graphic messages through interpreting, exemplifying, classifying, summarizing, inferring, comparing, and explaining. Applying is carrying out or using a procedure through executing, or implementing. Analyzing is breaking material into constituent parts, determining how the parts relate to one another and to an overall structure or purpose through differentiating, organizing, and attributing. Only the best of BSEE (Bachelor of Science in Electrical Engineering) students are able to truly attain the analysis level $[1,4]$. Evaluating is making judgments based on criteria and standards through checking and critiquing. Creating is putting elements together to form a coherent or functional whole; reorganizing elements into a new pattern or structure through generating, planning, or producing. The last two levels only grade students are able to attain them [1, 4].

Different initiatives by the IEEE Power and Energy Society (PES) and other professional organizations proposed to develop [10-13] a platform that simulate smart grids to be used in education, research and training. This upgraded laboratory helps senior-level undergraduate and graduate students learn and gain hands-on experience that prepares them to work in the area of smart grids. The laboratory will also provide interdisciplinary SG technical areas where working professionals and engineers will be trained. This laboratory also supports post-graduate and research scholars to contribute to the field of SG and build a strong collaboration with industry. The main features of the proposed laboratory upgrading can be summarized in the following:

- Development should be significant in terms of appropriate instructional design, assessment and evaluation tools.

- Integrating the use of IPad, laptops, software and ability to access technology to build an interactive/collaborative learning environment in- and outside classroom.

- Defining clear measures for assessment and monitoring the interaction levels among students and instructors.

- Updating course materials according to recent changes related to SG implementations and applied codes/standards.

- $\quad$ Provide a mean for marketing SG related courses to utilities, industrial and other educational sectors in the society.

The main objectives of the proposed laboratory upgrading are:

- Enhancing the design and delivery of the power system courses related to SG to meet the students' different learning styles.

- Utilizing new tools that cater the different learning modes and tendencies of students so as to increase their comprehension and retention of subjects covered.

- Re-designing interactive power system and SG related courses considering upgrading development in the laboratory.

- Defining different courses and/or learning modules that can benefit from the upgraded laboratory and provide needed insights into the integration concepts associated with smart grid applications.

Laboratory upgrading helps the students to gain a better understanding of the concept of smart grids and enhances students' practical skills and experience with actual Intelligent Electronic Devices (IEDs). Students will understand the measurement, monitoring, protection, control and communication features of IEDs. They will study and test other IEDs applications such as an energy analyzer, digital fault recorders, the sequence of event recorders, and smart energy meters. This upgrading will allow students to understand the concept of migrating to new technology while having the old technology functioning in order to compare existing electromechanical relays with the new IEDs. Furthermore, students will be able to observe and quantify the amount of operational and non-operational data that can be utilized before and after upgrading the PSS as well as the impact of this data on smart grid design and operation. Students will be able to understand the concept of communicating operational data to the SCADA (Supervisory Control and Data Acquisition) master station, nonoperational data to the enterprise data warehouse, and providing remote access capability. They will define 
and understand the implemented communication protocols in the smart grids.

At the research level, laboratory upgrading will provide a platform to simulate and analyze different SG research topics such as integrating renewable energy resources, micro grids, self-healing systems, synchrophasors, various communication, wireless sensor network applications, interoperability, cyberspace security, and more.

\section{EXISTING UAEU POWER System SimUlator}

The existing Hampden 180 Power System Simulator (PSS) is a modular system with seven discrete sections. It is available with manual controls for hands-on operation, as well as designed for integration with a customersupplied-computer. The Model 180 PSS is composed of actual power system components; relaying, metering, switching and remote data acquisition equipment [14]. Fig. 2 shows the front side and back side of the UAEU existing power system simulator.

The generation section has the apparatus for control of power generation, interconnection and synchronization of two or more systems. Outputs of two external $1.0 \mathrm{~kW}$, three-phase alternators are fed to the panel where provision is made for controlling the field excitation of each and its respective drive motor. An external generator (Section 2) can be integrated with the system using the available external terminal, monitoring instruments and reverse power relay. In the residential distribution section (Section 3) transformers step the transmission voltage down to distribution levels and distribution voltage down to utilization level. It contains residential loads, power consumption meters, temperature and current recording meters. Seven resistive loads represent residential demand. Timing system condenses a 24-hour cycle of loading into two real-time hours. Each pair of loads has $\mathrm{kWh}$ meter.

The industrial feeder (Section 4) has a voltage regulator, power factor correction capacitor bank and pf (power factor) meter, a recording ammeter, and a $\mathrm{kWh}$ meter. Power, heat and lighting loads are portrayed, each with the same timer as the residential loads. One of the industrial loads is inductive. Section 5 and 6 represent commercial distribution and substation operation. Section 7 has the required apparatus for interconnection to a second system. This include a demand type $\mathrm{kWh}$ meter, a three phase manual voltage regulator, a voltammeter and ammeter. A utility source $(\mathrm{G} 4)$ is interconnected with a dual bus that is tapped for transmission lines to generating stations on Sections 1 and 2, and for the feed lines to the distribution network on Section 5. Two directional relays are installed to study faults on the transmission lines. The PSS is designed to generate and study the following faults [14]:

- Line D1-11 line-to-line fault of $(25+\mathrm{j} 43.3 \mathrm{ohm})$ fault impedance on Section 6 to demonstrate direction overcurrent relays on Section 2 and Section 7.

- Substation 4 internal transformer line-to-line fault of $100 \mathrm{ohm}$ to demonstrate transformer differential relays in Section 3.

- Industrial feeder fault line-to-line fault of $100 \mathrm{ohm}$ at regulator primary in Section 4 to demonstrate transformer overcurrent relays, industrial feeder overcurrent relays and transformer differential relays.
- External generator (Gen. 3) phase reversal to demonstrate generator synchronizing.

- Network reverse power in Section 5 to demonstrate network reverse power relay.

- Phase reversal in Section 6 to demonstrate reverse phase relay.

The single line diagram (SLD) of the PSS is presented in Fig. 3. The system has different operation scenarios. Generators 1, 2 and 3 can be synchronized and operate in parallel. Generator 3 is external generator which can be of renewable energy type integrated with the whole simulator or supply part of the load to simulate the microgrid. Generator 4 is the utility supply which also can be integrated with the simulator generators through the transmission lines or underground cables. The simulator switches and circuit breakers can be managed to provide different system configurations of generation, transmission and distribution to supply residential, industrial, agriculture and substation services loads [14].

The simulator data under normal operation condition and during simulated faults and/or switching conditions is investigated for the setup of the new integrated IEDs. Samples of momentary fault condition currents in circuit breaker 106 (Generator 2) and bus-section circuit breaker 305 are presented in Fig. 4.



(a)



(b)
Figure 2. Hampden 180 Power System Simulator a- front side and bback side

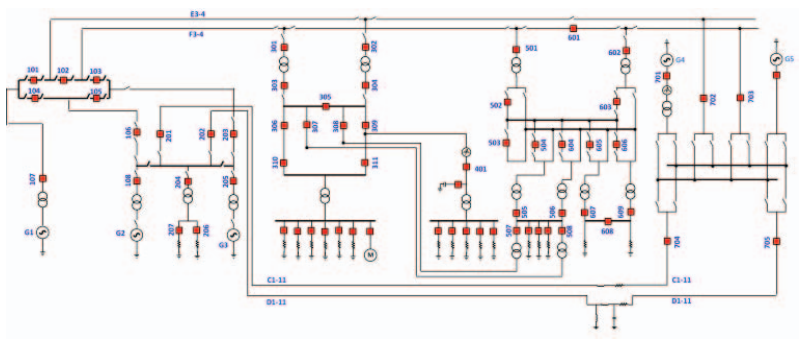

Figure 3. Single line diagram of Hampden 180 Power System Simulator
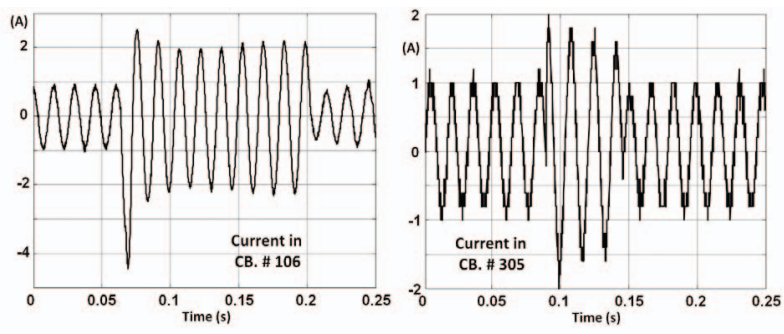

Figure 4. Momentary Fault Condition Currents in Hampden Simulator 


\section{UPGRADING UAEU POWER SYSTEM SIMULATOR}

The Smart grid laboratory is upgraded by integrating the IEDs, satellite-synchronized clock, real-time automation controller, Ethernet switch, embedded automation computing platform and wireless sensors within the Hampden 180 existing power system simulator (PSS). The following upgraded elements are supplied with IEC-61850 communication protocol for substation control and monitoring:

- SEL-700G Generator Protection Relay

- SEL-411L Advanced Line Differential Protection, Automation, and Control System

- SEL-787E Transformer Protection Relay

- SEL-487B Bus Differential and Breaker Failure Relay

- SEL-710 Motor Protection Relay

- SEL-751A Feeder Protection Relay

- SEL-451 Protection, Automation, and Bay Control System

- $\quad$ SEL-2407 Satellite-Synchronized Clock

- SEL-3530 Real Time Automation Controller

- $\quad$ SEL-2730M Managed 24-Port Ethernet Switch

- SEL-3354 Computing Platform

- AMS-4000 Relay Test System

Fig. 5 shows the single line diagram of the upgrading stages and photos of the constructed IEDs panels. The main features of the upgraded elements are their ability to measure, monitor, control, protect, store and communicate data. These features can serve the main areas of smart grids and summarized as the high-speed of protection and the capability of accurately pinpointing faults using GPSsynchronized traveling wave fault location $[15,16]$. The IEDs can be utilized to view synchrophasors and measure the system state, monitor circuit breaker performance. The IEDs can monitor the number of through faults, accumulated $I^{2} t$, and fault duration times to determine the frequency and impact of external faults on the transformers and other equipment. The flexibility and scalability of the relays can be used to protect a wide variety of bus configurations, including: Single bus, multifeeder, double-bus sections with tie breaker, two busbar, breaker-and-a-half configurations, double busbar and transfer busbar, HV and LV busbar feeders and three check zones as trip supervision $[15,16]$.

The Embedded Automation Computing platform (SEL3354) has the ability to collect data from IEDs and extract information. It can synchronize the time clocks and handle communications and information sets for large and small substation integration projects. Satellite-Synchronized Clock with high accuracy provides accurate timing signals to phasor measurement units and protective relays to assure event correlation to the absolute accuracy provided by the relay $[15,16]$.

Furthermore, education in smart grid field could be enhanced by enabling students to do capstone projects that are related to interfacing the traditional power system elements with intelligent wireless sensors networks. Student would acquire a number of skills that is crucial in the new field of SG. The United States Department of Energy (DOE) expects that the widespread deployment of wireless sensor networks (WSN) in industry could improve overall production efficiency by $11 \%$ to $18 \%$ and, in addition, reduce industrial emissions by more than $25 \%$ [17, 18]. IEEE 802.11 (Wi-Fi), IEEE 802.15.1 (Bluetooth), IEEE 802.15.3 (UWB), and IEEE 802.15.4 (ZigBee) are possible candidates for integrating sensors with wireless technologies in order to provide reliable data and to transform existing power systems into smart grids.

The nature of the acquired skills is interdisciplinary, ranging from embedded system design to software design. An example of the previous statement could be interfacing a low cost microcontroller which has a wireless communication capability to the current and voltage transformers in a distribution substation as shown in Fig.6. Such a project would require developing the embedded hardware design as well as the software design.

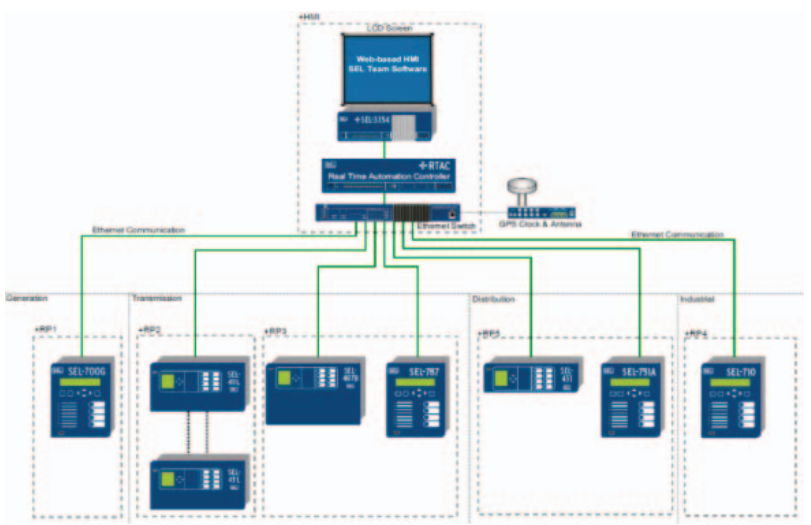

(a)

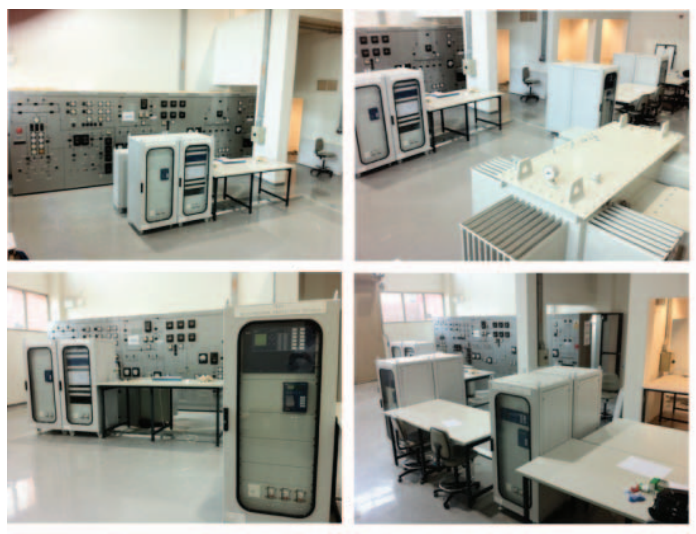

(b)

Figure 5. . a- Single line diagram of the IEDs panels and Ethernet connections, b- Hampden simulator and the IEDs panels.

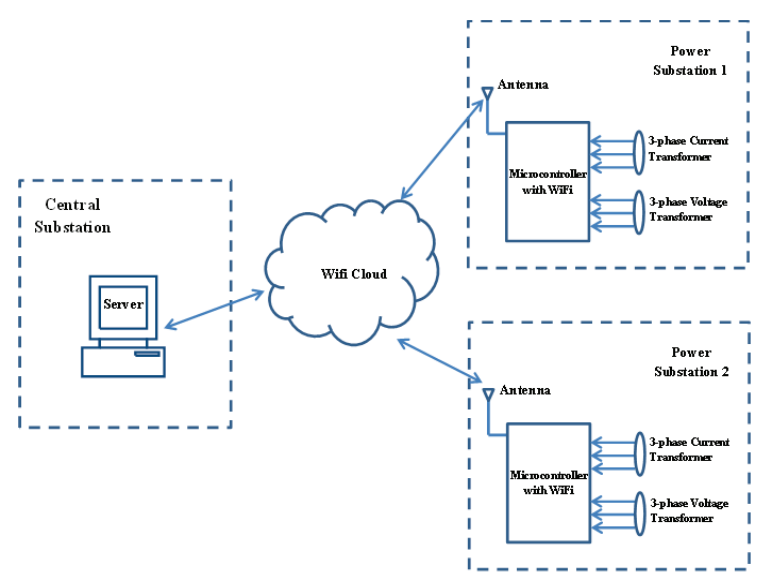

Figure 6. Wireless Sensor based Educational Project 
Students would learn how to write programs on different platforms (PC, Tablets, and Smart phones) that would be interfaced with the traditional substation elements to monitor different signals and status (ie. Temperature of a transformer) which will provide a layer of safety for the monitoring purposes without direct contact with the substation equipment. Open source hardware and software implementation would be beneficial for the educational purposes to allow the students to add and improve the design of the hardware as well as the software.

A sample of a microcontroller board which could be used as an educational platform is the Arduino board (an open-source electronic prototyping platform) [19]. Different modules (shields) from different vendors could be added to the main board to add more capabilities to the system such as wireless communication. Fig. 7 illustrates Arduino Uno board and its technical specification is presented in Table 1.

The board would be interfaced with current and voltage transducers from the main current and voltage transformers in each substation and fed into the analog inputs of the Arduino board to monitor the voltage and current in all the phases and detect faults [6]. If a fault is detected the information would be sent through a WiFi network to a central substation in order to locate the site of the fault. The central substation would have prior information about the topology of the distribution network. Each substation would be assigned an address that would be sent through WiFi along with fault status information $[20,21]$.

\section{EDUCATIONAL OUTCOME}

This upgrading will give students the opportunity to experiment with the new technologies and simulate smart grids in the laboratory. The following are some of the expected learning enhancement and educational outcomes expected from the new development:

- The upgraded system will demonstrate high level of functionality and performance while satisfying the operational and design objectives of smart grid. Students will enhance their experimental ability to measure, monitor, control, protect, store and communicate data.

- Understand the concept of migrating to new technology while having the old technology functioning (Electromechanical relays and $\mathrm{kWh}$ meters) in order to experiment and compare the impact of technology deployment in transforming conventional systems toward smart grids.

- Discuss, employ and analyze network-based communication technologies, including Ethernet, IEC 61850 and SCADA protocols. This system-wide, networkbased communication allow contact with all protection, control and monitoring devices and boosts a variety of smart grid applications such as smart monitoring, centralized control, distributed peer-to-peer control and wide-area protection.

- Practice, experiment and analyze information processing at IEDs device level and data sharing and processing among networked IEDs at system level to extract and create power system information. This information is vital to smart grid tasks that include both mission-critical protection and control applications.

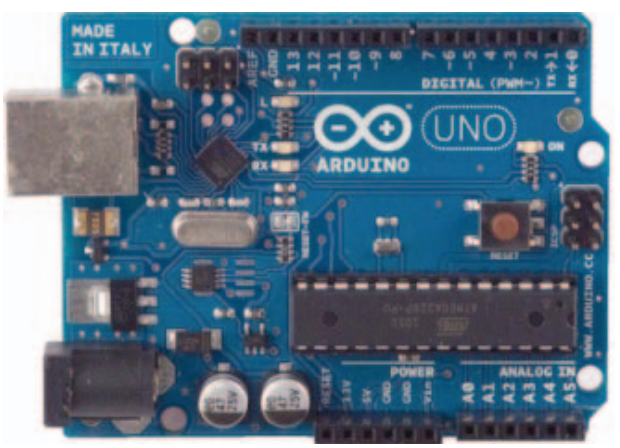

Figure 7. Arduino Uno board

TABLE 1.

TECHNICAL SPECIFICATION OF THE ARDUINO UNO BOARD

\begin{tabular}{|c|c|c|c|}
\hline Microcontroller & Atmega328 & $\begin{array}{c}\text { DC Current } \\
\text { per I/O Pin }\end{array}$ & $40 \mathrm{~mA}$ \\
\hline $\begin{array}{c}\text { Operating Volt- } \\
\text { age }\end{array}$ & $5 \mathrm{~V}$ & $\begin{array}{c}\text { DC Current for } \\
\text { 3.3V Pin }\end{array}$ & $50 \mathrm{~mA}$ \\
\hline Input Voltage & $7-12 \mathrm{~V}$ & Flash Memory & $32 \mathrm{~KB}$ \\
\hline $\begin{array}{c}\text { Input Voltage } \\
\text { Limit }\end{array}$ & $6-20 \mathrm{~V}$ & SRAM & $2 \mathrm{~KB}$ \\
\hline Digital I/O Pins & 14 & EEPROM & $1 \mathrm{~KB}$ \\
\hline $\begin{array}{c}\text { Analog Input } \\
\text { Pins }\end{array}$ & 6 & Clock Speed & $16 \mathrm{MHz}$ \\
\hline
\end{tabular}

- Upgrading functions such as sequential event recording, digital fault recording, transformer, breaker and battery conditions monitoring will allow students to generate comprehensive reporting to diagnose events, schedule knowledge-based maintenance, detect abnormal trends, and satisfy information requirements of supervisory systems.

- The students will be able to define, discuss and evaluate the threats against vulnerable system components and apply information security.

- The students will be able to interface the traditional power system elements with intelligent wireless sensors networks. They will write programs on different platforms (PC, Tablets, and Smart phones) that would be interfaced with the traditional substation elements to monitor different signals and status.

In addition, the available external terminal of generator 3 might be utilized to integrate a renewable energy source with Hampden simulator and illustrate the challenges of integrating renewable energy resources and the development of microgrids.

\section{CONCLUSION}

The paper introduces stage 1 of upgrading the UAEU power system laboratory towards teaching the smart grids. The satellite-synchronized clock, real-time automation controller, Ethernet, embedded automation computing platform and wireless sensors are installed and integrated with the conventional Hampden 180 simulator. The upgraded laboratory is designed to demonstrate high level of functionality and enhance students' experimental ability, and understand the concept of migrating to new technology.

Students will have the ability to practice an integrated solution of network-based communication technologies, including Ethernet, IEC 61850 and traditional SCADA protocols. They will practice information processing at 
device level and at system level and extract important information for protection, control and asset management. The students will be able to define, discuss and evaluate the importance of cyber security to overcome the threats against vulnerable system components.

\section{ACKNOWLEDGMENT}

Authors would like to thank the Emirates Foundation for their support to upgrade the UAE University power system laboratory. This work is financially supported by the Emirates Foundation under a contract no. \# 2010/179.

\section{REFERENCES}

[1] Gerald T. Heydt, Anjan Bose, Ward T. Jewell, Mladen Kezunovic, James D. McCalley, Dennis J. Ray,Peter W. Sauer, Chanan Singh, and Vijay Vittal, "Professional Resources to Implement the "Smart Grid" North American Power Symposium(NAPS), 2009, PP. 1-8.

[2] Farag Sallabi, A. M. Gaouda, A. H. El-Hag and M.M.A. Salama, "Evaluation of ZigBee Wireless Sensor Networks Under High Power Disturbances," in press 2013, IEEE Transactions on Power Delivery.

[3] Abiodun Iwayemi, Peizhong Yi, Peng Liu, and Chi Zhou, "A Perfect Power Demonstration System," Innovative Smart Grid Technologies (ISGT), Gaithersburg, MD,19-21 Jan. 2010

[4] M. Kezunovic,'Teaching the Smart Grid Fundamentals Using Modeling, Simulation, and Hands-on Laboratory Experiments," Power and Energy Society General Meeting, IEEE 2010, PP. 1-6.

[5] Stuart Borlase, Smart Grids: Infrastructure, Technology, and Solutions, CRC Press, 2012.

[6] J. Ren, M. Kezunovic "Modeling and Simulation Tools for Teaching Protective Relaying Design and Application for the Smart Grid," Modern Electric Power System (MEPS), Proceeding of International Symposium, 2010, PP. 1-6.

[7] M. Kezunović, "A Survey of Engineering Tools for Protective Relaying,” ELECTRA, No. 225, pp 26-30, April 2006.

[8] David R. Krathwohl, A Revision of Bloom's Taxonomy: An overview, THEORY INTO PRACTICE, College of Education, The Ohio State University Volume 41, Number 4, Autumn 2002.

[9] Benjamin Bloom, Taxonomy of Educational Objectives, Handbook I: The Cognitive Domain, David McKay Co., New York, NY, 1956.

[10] Noel N. Schulz, Senior Member, IEEE, "Integrating Smart Grid Technologies into an Electrical and Computer Engineering Curriculum," Innovative Smart Grid Technologies ASIA (ISGT), IEEE PES 2011, PP. 1-5.
[11] M. Shahidehpour, Zuyi Li Illinois Institute of Technology,"A World-Class Smart Grid Education and Workforce Training Center," Power and Energy Society General Meeting, IEEE 2010.

[12] Anurag K Srivastava, Senior Member, IEEE, Carl Hauser, David Bakken, Senior Member, IEEE, and Min Sik Kim, Member, IEEE," Design and Development of a New Smart Grid Course at Washington State University," Power and Energy Society General Meeting, IEEE 2012, PP. 1-2.

[13] Gregory F. Reed, Member, IEEE; William E. Stanchina, Member, IEEE, "Smart Grid Education Models for Modern Electric Power System Engineering Curriculum," Power and Energy Society General Meeting, IEEE 2010, PP. 1-5.

[14] Hampden Engineering Corporation, Student Exercises for the Hampden 180 Power System Simulator, Bulletin 180-EX-ED3.

[15] Hector J. A. Ferrer and Edmund O Schweitzer, Modern Solutions for Protection, Control and Monitoring of Electric Power Systems, Schweitzer Engineering Laboratory Inc. 2010.

[16] Alstom Grid, Network Protection and Automation Guide, Protective Relays Measurements and Control, Alstom, 2011.

[17] Bin Lu, Thomas G. Habetler, and Ronald G. Harley, "A Novel Motor Energy Monitoring Scheme using Wireless Sensor Networks," Industry Applications Conference, the 41st IAS Annual Meeting, Oct. 2006.

[18] Department of Energy, "2002 industrial wireless technology for the 21st century," Office of Energy Efficiency and Renewable Energy Report, Department of Energy, Washington D.C., 2002.

[19] SparkFun Electronics, https://www.sparkfun.com/categories

[20] Bin Lu, Thomas G. Habetler, and Ronald G. Harley, "A Novel Motor Energy Monitoring Scheme using Wireless Sensor Networks," Industry Applications Conference, the 41st IAS Annual Meeting, Oct. 2006.

[21] Department of Energy, "2002 industrial wireless technology for the 21st century," Office of Energy Efficiency and Renewable Energy Report, Department of Energy, Washington D.C., 2002.

\section{AUTHORS}

A. M. Gaouda, Ahmed Abd-Rabou and Abdulrahman Dahir are with UAE University, Al Ain, UAE.

This article is an extended and modified version of a paper presented at the 2013 IEEE International Conference on Teaching, Assessment and Learning for Engineering (TALE2013), held 26-29 August 2013, Bali Dynasty Resort, Kuta, Indonesia. Submitted 10 September 2013. Published as re-submitted by the authors 09 March 2014. 